\title{
THE STRUCTURE OF GENERALIZED ACCESSIBLE RINGS
}

\author{
BY ERWIN KLEINFELD, MARGARET HUMM KLEINFELD \\ AND J. FRANK KOSIER ${ }^{1}$
}

Communicated by I. N. Herstein, October 9, 1968

In [3] Schafer has defined generalized standard rings as rings satisfying the identities

$$
\begin{aligned}
(x, y, x) & =0 \\
\text { (1) } & \\
\text { (2) } & (x, y, z) x+(y, z, x) x+(z, x, y) x=(x, y, x z)+(y, x z, x)+(x z, x, y)
\end{aligned}
$$$$
\text { (3) }(x, y, w z)+(w, y, x z)+(z, y, x w)=(x,(w, z, y))+(x, w,(y, z))
$$

and observed that these identities imply

$$
(y, y,(x, z))=0
$$

and for characteristic not three

$$
\left(x, y, x^{2}\right)=0 .
$$

Schafer determines the structure of simple, finite-dimensional generalized standard algebras of characteristic not 2 or 3 by showing that they must be either commutative Jordan or alternative.

Previously one of the authors [2] had studied accessible rings, which are defined by the identities

$$
(x, y, z)+(z, x, y)-(x, z, y)=0
$$

and

$$
((w, x), y, z)=0 .
$$

The structure of accessible rings is determined in that paper as it turns out that an accessible prime ring must be either associative or commutative.

Both of these results generalize some results on standard algebras by Albert [1].

In the present announcement we define an even more general class of rings called generalized accessible, as those satisfying the identities (8)

$$
(x,(z, y, y))=0 \text {, }
$$

1 This work was supported in part by a Grant from the U.S. Army Research Office (Durham), and the National Science Foundation NSF Grant GP-6365. 
(9) $3(x, y,(w, z))=-(w,(x, y, z))-2(x,(y, z, w))$

$$
+2(y,(z, w, x))+(z,(w, x, y))
$$

and (1). The following schematic diagram shows that generalized accessible is implied by commutative as well as by alternative, and also by identities (1) and (3).

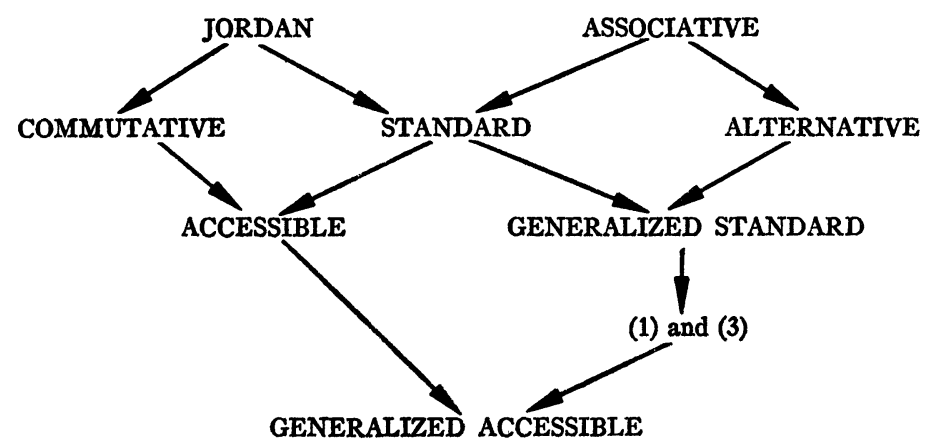

Our main result is the following:

MAIN Theorem. Let $R$ be a prime generalized accessible ring of characteristic $\neq 2,3$. Then $R$ must be either alternative or commutative.

From this result one obtains immediately all the previous results on prime accessible, generalized standard, as well as standard rings. Moreover the usual extension of the Jacobson radical (as in the alternative case) leads to a similar result for semisimple rings.

We shall present here an outline of the proof of the Main Theorem. Details will appear elsewhere.

LEMMA 0. (1) and (3) imply the following:

(i) $(x,(z, y, y))=0$,

(ii) $(x, y,(w, z))+(x, w,(z, y))+(x, z,(y, w))=2(x,(w, z, y))$,

(iii) identity (9).

Thus, a ring satisfying (1) and (3) is generalized accessible and clearly any accessible ring is generalized accessible.

From now on we assume $R$ is a generalized accessible ring of characteristic $\neq 2,3$.

LEMMA 1. $((w \sigma, x \sigma, y \sigma), z)=\operatorname{sgn} \sigma((w, x, y), z)$ where $w, x, y$, and $z$ are arbitrary in $R$ and $\sigma$ is any permutation of the three elements $w, x$, and $y$.

Definition. We say elements $x, y$ and $z$ of $R$ form an alternative 
triple in case for every permutation $\sigma$ on three letters $(x \sigma, y \sigma, z \sigma)$ $=\operatorname{sgn} \sigma(x, y, z)$.

LEMMA 2. The elements $x, y$ and $z$ of $R$ form an alternative triple provided at least one of them is a sum of commutators.

LEMMa 3. Let

$H(x, y, z)=(x, y, z)+(y, z, x)+(z, x, y), S=\{s \in R \mid(s, R)=0\}$

and $C$ equal the center of $R$. Then

(i) $H(x, y, s)=0$,

(ii) $(s \sigma, x \sigma, y \sigma) \in S$,

(iii) $S$ is a subring of $R$,

(iv) $(x \sigma, y \sigma, z \sigma)=0$ provided one element is in $S$ and another is a sum of commutators,

(v) $S \cap(R, R) \subset C$.

LEMMA 4. If $V=\sum(R, R)$ then $Q=V+V R$ is an ideal of $R$.

LEMMA 5. If $U=\{u \in S \mid u V=0\}$ then $U$ is an ideal of $R$ such that $U Q=0$.

LEMma 6. $(S, y, y) V \subset S$.

LEMma 7. $(S, R, R) V \subset S$.

In the next two lemmas $R$ is assumed to have no trivial ideals.

LEмma 8. If $s \in S, s V \subset S$, then $s \in U$.

LEMMA 9. If $(S, R, R)=0$, then $R$ is alternative.

From these lemmas the proof of the main theorem is now obvious; for if $R$ is prime, then it follows from Lemma 5 that either $Q=0$ so that $R$ is commutative or that $U=0$. Let us suppose $R$ is not commutative. Then by Lemmas 3 and 7 it follows that the elements of $(S, R, R)$ satisfy the hypothesis of Lemma 8 , so that $(S, R, R) \subset U=0$. But then Lemma 9 implies that $R$ must be alternative.

\section{REFERENCES}

1. A. A. Albert, Power associative rings, Trans. Amer. Math. Soc. 64 (1948), 552593. 340.

2. Erwin Kleinfeld, Standard and accessible rings, Canad. J. Math. 8 (1956), 335-

3. R. D. Schafer, On generalized standard algebras, Proc. Nat. Acad. Sci. U.S.A. 60 (1968), 73-74.

UNIVERSITY of Iowa, Iowa City, Iowa 52240 